\title{
Intrusions of surface sewage plumes into continental shelf waters: interactions with larval and presettlement juvenile fishes
}

\author{
Charles A. Gray* \\ NSW Fisheries Research Institute, PO Box 21, Cronulla, New South Wales 2230, Australia
}

\begin{abstract}
Primary treated sewage (domestic and industrial) is discharged through several shoreline and deepwater outfalls into the coastal waters off Sydney, southeastern Australia. The effluent discharged from the shoreline outfalls formed highly visible surface plumes that intruded up to $5 \mathrm{~km}$ seaward and $8 \mathrm{~km}$ along the coast from their point of discharge. Each sewage plume was a lens ( 1 to $5 \mathrm{~m}$ deep) of low-salinity turbid water that overlay high-salinity clear shelf water, and distinct frontal regions usually developed between plume and shelf water. Large numbers of young fishes in surface waters were caught in and around the sewage plumes. In particular, many fish were concentrated at the fronts (e.g. Carangidae, Engraulididae, Sparidae), which was probably the result of advection at fronts as well as behavioural responses of fish. Surface sewage plumes therefore affected small-scale $(<1 \mathrm{~km})$ patterns of distribution and density of young fishes. The oceanography of sewage plumes may increase and prolong exposure of fishes to pollutants during early development. Sewage plumes could affect young fishes spawned near to and at great distances from outfalls because of alongshore currents, and effects on fish populations could be manifest over large spatial scales. The influences of sewage plumes on larger-scale patterns of distribution, transport and survival of young fishes are discussed.
\end{abstract}

KEY WORDS: Sewage - Plumes - Oceanography · Young fishes - Distribution patterns

\section{INTRODUCTION}

Oceanographic features influence the distributions, densities and movements of small fishes and plankton in coastal waters (reviews in Haury et al. 1978, Owen 1981, Denman \& Powell 1984, Le Fèvre 1986, Kingsford 1990). Aggregations of fish larvae and plankton are often greatest at the convergence zones of different water bodies (Kingsford 1990, Govoni \& Grimes 1992, Govoni 1993) and are often manifest in surface waters. Larval fish and plankton have been found to be concentrated in and/or along the frontal regions of many oceanographic features, including: tidal, coastal and topographically controlled fronts and eddies (Epifanio 1987. Murdoch 1989, Kingsford et al. 1991); riverine and estuarine plumes (Mackas \& Louttit 1988, Gov-

•E-mail: grayc@fisheries.nsw.gov.au oni et al. 1989, Grimes \& Finucane 1991, Kingsford \& Suthers 1994); slicks associated with internal waves (Zeldis \& Jillett 1982, Shanks 1983, 1985, Kingsford \& Choat 1986). Material such as flotsam, drift algae and various pollutants can also be advected and concentrated around oceanographic features (Kingsford 1990, 1993, Brown et al. 1991, Tanabe et al. 1991, Kingsford \& Gray 1996).

The ways in which oceanographic features influence small fishes and plankton can vary depending on the type and magnitude of the oceanographic feature and according to the type, size and behaviour of the organism. For example, topographically controlled fronts and fine-scale currents can concentrate and retain small fish and plankton in a specific area (Alldredge \& Hamner 1980, Owen 1981, Wolanski \& Hamner 1988), whereas those entrained in large-scale eddies and the slicks associated with internal waves can be transported tens to hundreds of kilometres 
(Shanks 1983, Lobel \& Robinson 1988). Furthermore, small zooplankton may be passively advected into such oceanographic features, whereas small fishes may voluntarily aggregate in these features due to their invertebrate prey being concentrated there (Kingsford 1990). Consequently, oceanographic features can have substantial influence on the transport, survival and recruitment of fish and other meroplankton in coastal waters (Shanks 1983, Kingsford 1990, Grimes \& Finucane 1991).

In addition to the many natural oceanographic features, young fishes and plankton in estuaries and nearshore coastal waters may also be subjected to a wide variety of man-made intrusions, including breakwalls, groynes, wharves and various types of outfalls, which can interact with mainstream water bodies to create physical disturbances that behave in similar ways to natural oceanographic features. For example, eddies can form behind groynes and breakwalls like those behind headlands, islands and reefs (Emery 1972, Wolanski \& Hamner 1988, Murdoch 1989). The effluent released from different types of outfalls (e.g. sewage) often forms conspicuous plumes that have similar oceanographic attributes to natural (e.g. estuarine) plumes (Gray et al. 1992, Kingsford \& Gray 1996). Thus, man-made intrusions may potentially impinge on the dynamics of planktonic organisms. Moreover, effluent plumes from outfalls often contain large levels of toxic wastes (e.g. Duedall et al. 1983, Beder 1989), which can cause sub-lethal and lethal affects to young fishes and zooplankton (reviews in Rosenthal \& Alderdice 1976, Westernhagen 1988, Weis \& Weis 1989, Costello \& Read 1994, Kingsford \& Gray 1996). Effluent plumes are therefore a potential hazard to planktonic organisms.

Most field studies concerning the numerical influences of pollutants on planktonic fauna have either compared assemblages and/or densities of organisms near to and away from a polluted source (e.g. Hardy et al. 1987b, Karas et al. 1991, Nair et al. 1991. Gray et al. 1992), or have examined changes in fauna over a gradient of pollution from the point-source (e.g. Arfi et al. 1981). Few researchers have stratified sampling to account for biological effects of oceanography of pollution plumes on plankton. For example, Gray et al. (1992) compared densities of young fishes in 3 sewage plumes to non-plume (control) waters located some distance away $(>8 \mathrm{~km})$. They sampled in and below each plume, but found no consistent differences in densities of fishes in plume and non-plume waters. They hypothesised, however, that densities of fish may differ between water masses of the plume, front and adjacent ocean. Kingsford \& Gray (1996) found that densities of young fishes were often highest in the frontal edge of one sewage plume. The frontal hypothesis needs to be tested at several outfalls and potentially differential influences on different types and sizes of young fish need to be further examined. This paper documents how surface sewage plumes intrude into mainstream coastal currents off Sydney (Australia), and influence the distributions and densities of larval and presettlement juvenile fishes

\section{MATERIALS AND METHODS}

Sydney's sewage outfalls. Until 1991 sewage was discharged into the coastal waters off Sydney via 7 shoreline (cliff-face) outfalls. The distributions of young fishes in and around the effluent plumes associated with the point-source shoreline sewage outfalls at North Head, Bondi and Potter Point (New South Wales, Australia) (Fig 1) were examined herc. Each outfall discharged primary treated sewage effluent through a point-source into shallow subtidal waters $(2$ to $5 \mathrm{~m}$ ) at the base of the coastal cliffs. The effluent formed conspicuous discoloured and turbid surface plumes that intruded into the surrounding coastal waters (Gray et al. 1992, Kingsford \& Gray 1996; and see Fig. 1). The discharged effluent consisted of a cocktail of domestic and industrial wastes, and although freshwater was the major constituent of the discharge, it contained contaminants in solution and suspension, as well as varying concentrations of pathogens, bacteria and toxic substances (e.g. $\mathrm{Cd}$, $\mathrm{Cr}, \mathrm{Zn}, \mathrm{Hg}$ ) (Beder 1989). The catchment area, volume, rate and content of discharge of effluent differed among outfalls (Beder 1989, Fagan et al. 1992). The volume, rate and content of the effluent discharged from the sewage treatment plants also fluctuated considerably within and among days. The average dry weather discharge $\left(\mathrm{Ml} \mathrm{d}^{-1}\right)$ was 293 at North Head, 163 at Bondi and 37 at Potter Point. Wet weather flows were considerably greater than flows during dry weather. Note that the North Head and Bondi (and Malabar) cliff-face outfalls have now been decommissioned and replaced by extended deepwater outfalls (see Fagan et al. 1992, Otway et al. 1996).

Design and procedure oi sampling. Each outfall was sampled twice, once in December 1990 and once in January 1991. In December, samples were collected in 3 water masses near each outfall: in the effluent plume, along the frontal edge of the plume, and just outside (approximately $500 \mathrm{~m}$ ) the plume in clear shelf water. In January, sampling was done in each of the 3 water masses, but at 2 distinct locations at each outfall. The order in which each water mass was sampled at each outfall was selected randomly. 
All sampling was done in daylight. Sampling was done over a short time interval to try to catch the same types of fish in each sampling period. Three replicate $3 \mathrm{~min}$ surface (0 to $1 \mathrm{~m}$ ) tows were done in each water mass at all outfalls using a cylindrical/conical net with a $80 \mathrm{~cm}$ diameter mouth, and with $500 \mu \mathrm{m}$ mesh in the body and $250 \mu \mathrm{m}$ mesh in the collecting bag. Filtration efficiency of net was 1:5. The net was positioned at the front and to one side of the FRV 'Wobbegong' (a $7 \mathrm{~m}$ twin-hulled vessel) to avoid influences of the wake and bow waves on catches. The net was towed at $1 \mathrm{~m} \mathrm{~s}^{-1}$, and a flow meter was positioned in the mouth of the net to measure the amount of water filtered per tow (average volume per tow was $100 \mathrm{~m}^{3}$ ). Samples were preserved in $10 \%$ formalin/seawater immediately following capture, and after 2 wk transferred to $70 \%$ alcohol in the laboratory. All fishes were sorted from catches, identified to lowest taxonomic level possible, and counted using a binocular microscope Herein the term young fish refers to all fish caught in the sampling gear, excluding yolk-sac larvae.

Depth of water was 15 to $30 \mathrm{~m}$ around each outfall. The positions of plumes on each sampling date were mapped from the water and when available, from maps provided by the NSW (New South Wales) Beachwatch helicopter service. The temperature and salinity of water in and around each plume were determined by lowering a submersible data logger that measured depth, temperature and salinity from the surface to $2 \mathrm{~m}$ above the seabed. At Potter Point the data logger was also slowly towed at a depth of $1 \mathrm{~m}$ from the middle of the plume transversely across the front into clear shelf water to show the change in salunity at the front.

Analysis of data. Catches of fishes were standardised to number caught (density) per $100 \mathrm{~m}^{3}$ of water filtered. Data were transformed to $\ln (x+1)$ pror to statistical analysis.

Differences in the structure of the fish assemblages between plume, front and ocean waters were assessed using non-metric multidimensional scalıng and simılarity-based measures, following the procedures outlined in Field et al. (1982) and Clarke (1993). Twodimensional ordination plots were generated by multidimensional scaling of similarity matrices based on the Bray-Curtis similarity measure. One-way analyses of similarities (ANOSIM) were used to determine which assemblages differed.

Density data and the derived variables (e.g. number of total taxa) were analysed by 2 - and 3-factor analyses of variance (ANOVA) Each sampling period was analysed separately. December-Outfall (random) and Water mass (fixed); January-Outfall (random), Location (nested in Outfall-random) and Water mass (fixed). Data were checked for homogeneity of varlances (Cochran's test) before analysis Heterogeneous data were still analysed because large and balanced data sets are robust to deviations from homogeneity, but alpha was set at $p=0.01$ to reduce the rusks of Type 1 errors (Underwood 1981) 


\section{RESULTS}

\section{Oceanography of surface sewage plumes}

Each sewage plume was a lens of discoloured, turbid, low-salinity water 1 to $5 \mathrm{~m}$ deep overlaying clear high-salinity shelf water (Fig. 2). Salinity of water in each plume was generally 1 to 5 pt less compared to the shelf waters surrounding and below each plume (Figs. 2 \& 3). The plumes usually formed distinct fronts with the surrounding ocean (Fig. 3), which were observed as distinct changes in salinity, turbidity and colour (see also Kingsford \& Gray 1996).

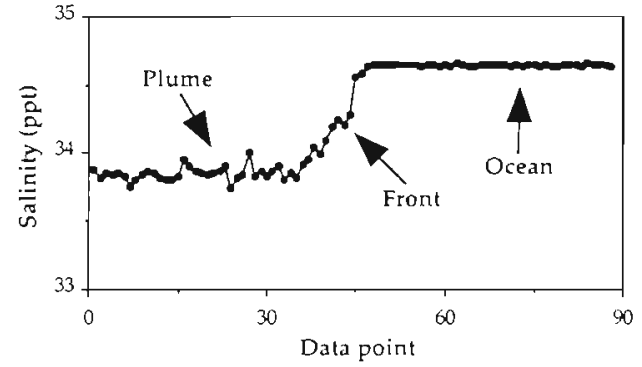

Fig. 3. Cross-section salinity structure of water column at $1 \mathrm{~m}$ depth across Potter Point sewage plume on 26 January 1991 Note the reduced salinity in the plume, and the abrupt change in salinity at the front between plume and ocean waters

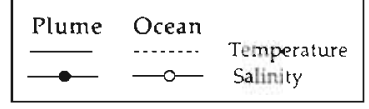

a) December
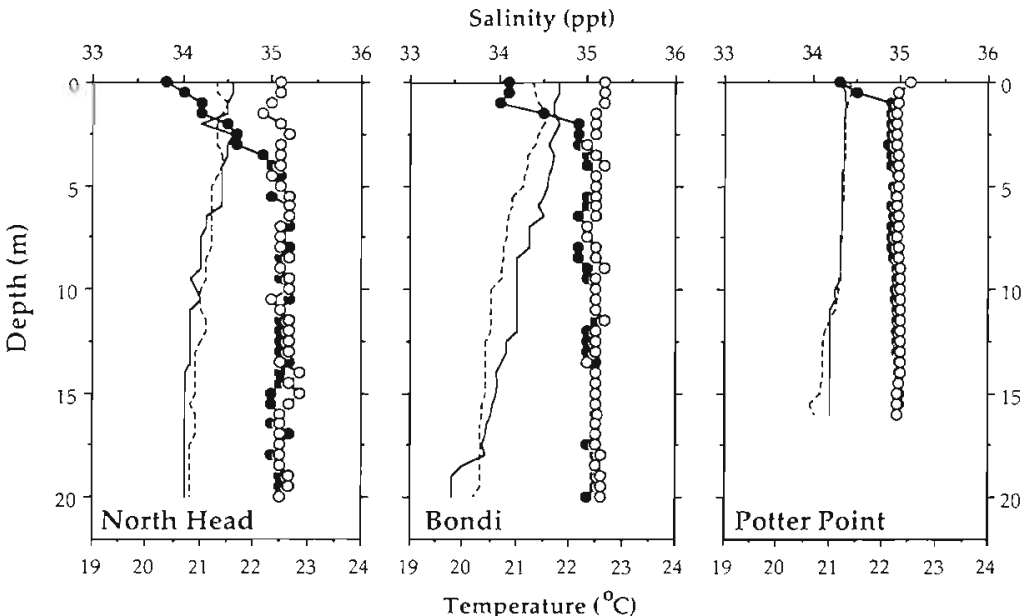

b) January
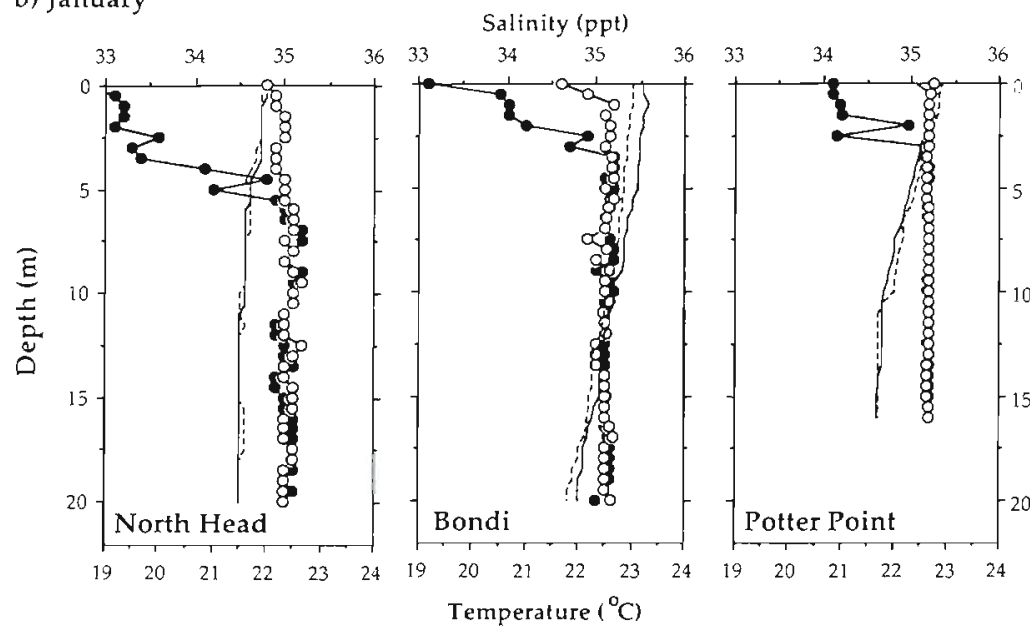

Fig. 2. Salnity and temperature profiles of the water column in and below the North Head, Bondi and Potter Point sewage plumes and in adjacent ocean waters. Note that the salinity of water was reduced by 1 to $3 \mathrm{ppt}$ in the top 2 to $3 \mathrm{~m}$ in the plumes compared to adjacent ocean waters
The size, shape and orientation of each plume differed between outfalls and between sampling periods (Fig. 1). Essentially, each plume was generally orientated alongshore, trailing downstream in the prevailing (predominantly southerly) current from the point of discharge. All 3 plumes flowed in a southerly direction during both sampling periods, except North Head in December. The North Head plume was generally the largest, followed by Bondi and Potter Point. The North Head and Bondi plumes extended between 1 and $3 \mathrm{~km}$ offshore and between 3 and $5 \mathrm{~km}$ alongshore, whereas the plume at Potter Point was more confined to Bate Bay (particularly in December), but extended about $3 \mathrm{~km}$ alongshore in January (Fig. 1). In each period the front was easily distinguished at all 3 plumes, except at Potter Point in December The front of each plume usually degenerated with increasing distance from the actual outfall. In each period a southerly current predominated (except at North Head in December), the swell was generally $<1 \mathrm{~m}$ and from either the northeast or southeast, whereas the wind was generally light $\left(<10 \mathrm{~m} \mathrm{~s}^{-1}\right)$ and from the northeast or southeast.

\section{Differences in assemblages of young fishes between water masses}

A total of 11328 fish of 49 families was caught in water surrounding the 3 outfalls (Table 1). Overall, most fish were caught at fronts and least in ocean 


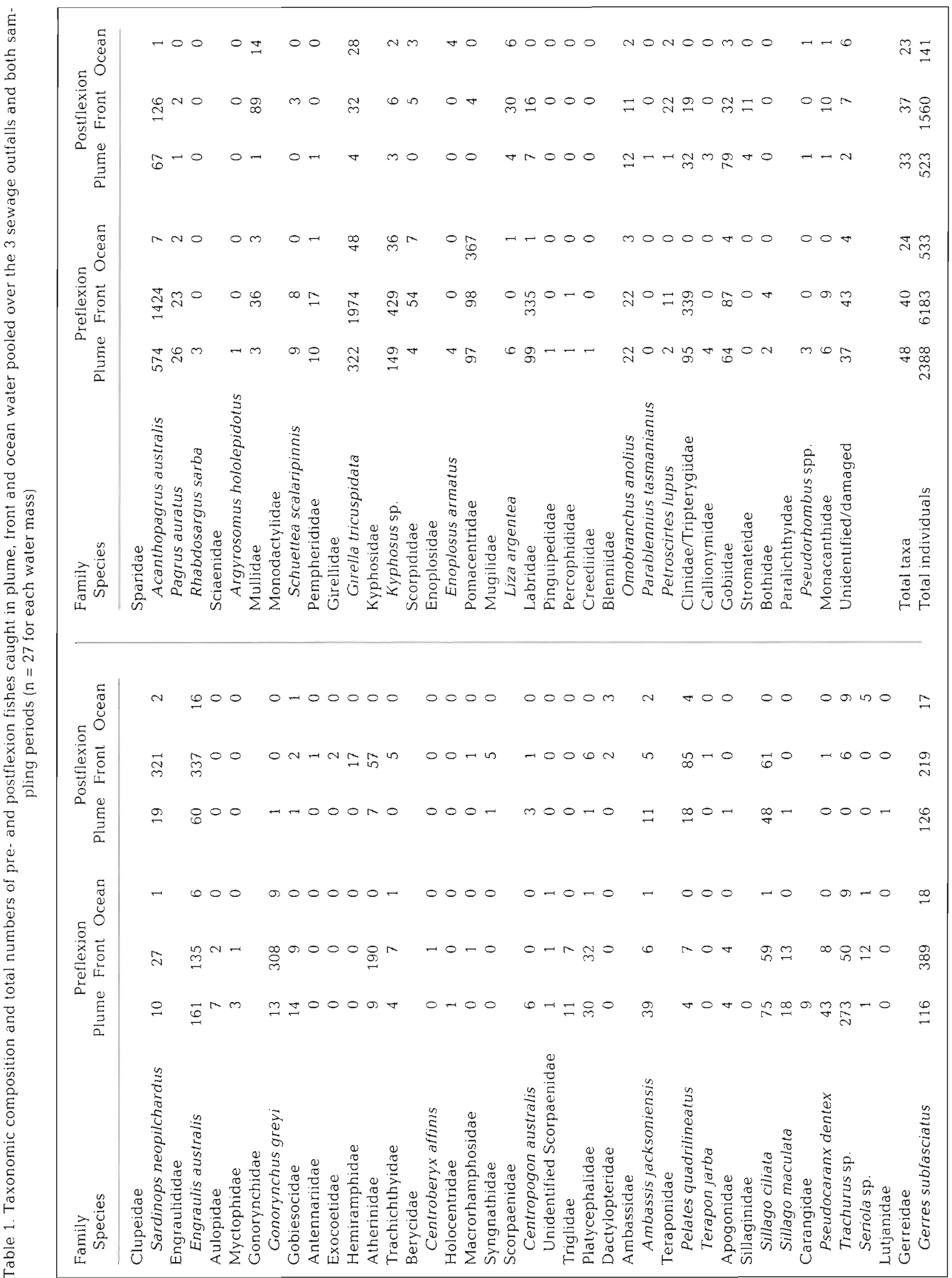


water. Taxonomic composition of samples varied spatially and temporally. Assemblages in plumes and fronts were most similar and differed to those in adjacent shelf water (Fig. 4, Table 2). However, at Potter Point differences among assemblages in plume, frontal and ocean waters were not clear as few fish were caught.

\section{Differences in densities of young fishes between water masses}

In both sampling periods greatest densities of fish were found in the plume or front at North Head and Bondi, but there were no clear patterns at Potter Point (Fig. 5, Tables 3 \& 4). This same pattern was also evident for most individual taxa, notably Acanthopagrus australis, Gerres subfasciatus, Engraulis australis, Girella tricuspidata, Kyphosus sp., Sardinons neopilchardus, Pelates quadrilineatus, Liza argentea, Omobranchus anolius, Petroscirtes lupus, Atherinidae, Trachurus sp., Labridae, Gobiidae, Sillago ciliata, Clinidae/Tripterygiidae, Mullidae, Pomacentridae, Platycephalidae (Tables 3-8). In December few fish were caught at Potter Point, but in January most Gobiidae, Atherinidae, Clinidae/Tripterygiidae, Platycephalidae, Pagrus auratus and Ambassis jacksoniensis occurred in the plume or front at Potter Point (Table 8).

Differences in densities of fishes between water masses in January were not always the same at both locations at each outfall [significant $\mathrm{L}(\mathrm{O}) \times \mathrm{W}$ in Table 4]. For example, densities of Engraulis australis and Sillago ciliata at North Head were greatest in the plume at Location 1 , but at the front at Location 2 (Table 6). Similarly, at Potter Point, greatest numbers of taxa were in the plume at Location 1 , but in ocean water at Location 2 (Table 8). Several taxa displayed consistent trends in densities between water

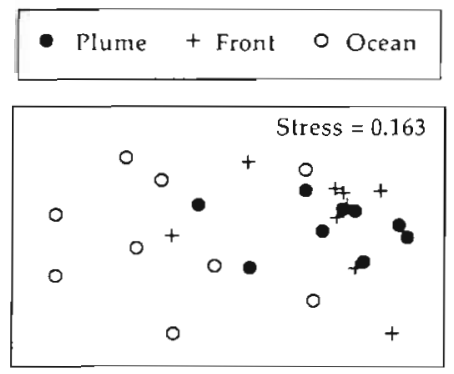

Fig. 4. Non-metric multidmensional scaling ordination plot displaying the relationships among the assemblages of larva] fishes in plume, front and ocean waters. Replicate data pooled for each water mass in December and January. Data transformed to $\log (x+1)$
Table 2 Summary of 1 -way analysis of similarities and pairwise comparisons of fish assemblages in plume, front and ocean waters. Replicate data pooled for each site, and both periods combined. 5000 permutations were used for each test. Data transformed to $\log (x+1)$. $\cdots, \cdots$ : significant at $p<0.01, p<0.001$, respectıvely; ns: not significant at $p>0.05$

\begin{tabular}{|lccc|}
\hline Effect & $\mathrm{R}$ & Sample size & Signuficance \\
\hline Treatment & 0.211 & 5000 & $\ldots$ \\
Plume vs Front & -0.028 & 5000 & $\mathrm{~ns}$ \\
Plume vs Ocean & 0.381 & 5000 & $\ldots$ \\
Front vs Ocean & 0.322 & 5000 &.. \\
\hline
\end{tabular}

masses at some outfalls, including Atherinidae and Mullidae which were most abundant at the front at both locations at North Head and Bondi (Tables $6 \& 7$ ).

Rank differences in densities of many fish among water masses at each outfall varied between periods. For example, there were no significant differences in the densities of Kyphosus sp. among water masses at North Head in December, but more were caught at the front in January (Tables $5 \& 6$ )
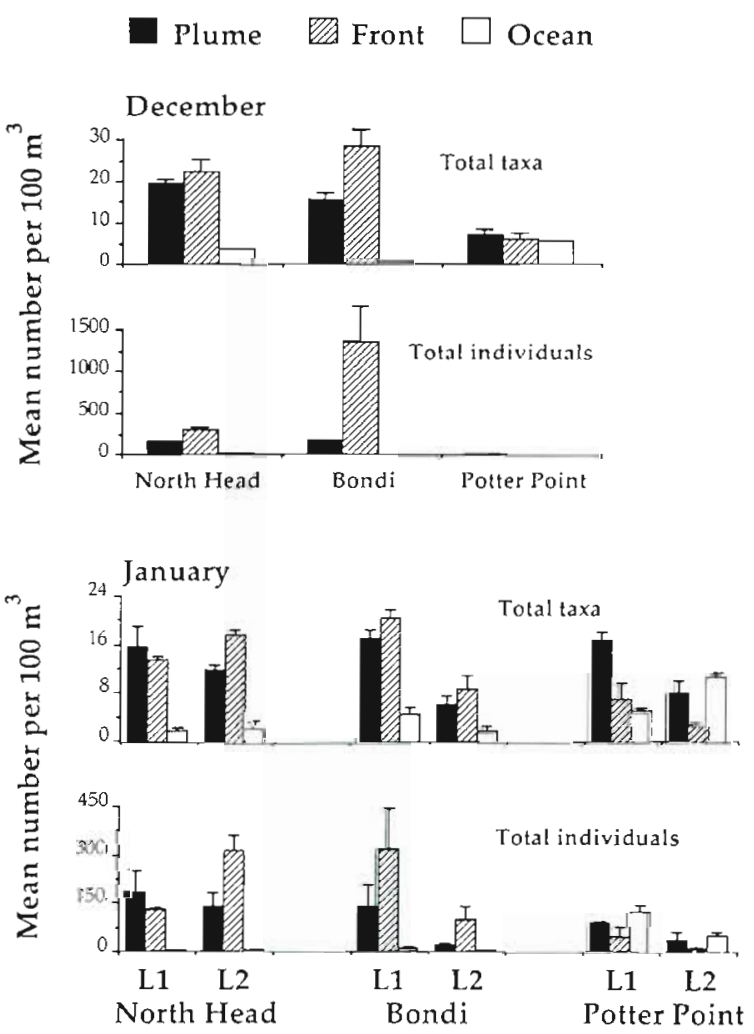

Fig. 5. Mean (+1 SE) numbers of total taxa and total individuals caught in plume, front and ocean waters at the North Head, Bondi and Potter Point sewage outfalls in December 1990 and January 1991. January: L1 = Location 1; L2 = Location 2 
Table 3. Summary of ANOVAs of the numbers of total taxa, total individuals and individual taxa caught in plume, front and ocean water at North Head, Bondi and Potter Point sewage outfalls in December 1990. Mean square values are shown. Data transformed to $\ln (x+1)$. Significance: $p<0.05, \cdots p<0.01, \cdots p<0.001$; ns: not significant at $p>0.05 ;$ CT: Cochran's test

\begin{tabular}{|c|c|c|c|c|c|}
\hline \multirow[t]{3}{*}{ Taxon } & \multicolumn{4}{|c|}{ Source of variation } & \multirow[t]{3}{*}{$\mathrm{CT}$} \\
\hline & Outfall & Water mass & $\mathrm{O} \times \mathrm{W}$ & Residual & \\
\hline & $(2,18)$ & \multicolumn{3}{|c|}{ Degrees of freedom in F-test } & \\
\hline Total taxa & $0.819 \cdots$ & $5.774 \mathrm{~ns}$ & $1.537^{\cdots}$ & 0.076 & ns \\
\hline Total individuals & $12.250 \cdots$ & $22.763 \mathrm{~ns}_{\mathrm{s}}$ & $7.550 \cdots$ & 0.234 & ns \\
\hline Acanthopagrus australis & $21.560 \cdots$ & $23.107 \mathrm{~ns}$ & $5.836^{\cdots} \cdot$ & 0.146 & $\cdot$ \\
\hline Ambassis jacksonıensis & $1.436 \cdots$ & $1.138^{\circ}$ & $0.080 \mathrm{~ns}$ & 0.173 & $\cdot$ \\
\hline Atherinidae & $1.105 \mathrm{~ns}$ & $8.213 \mathrm{~ns}$ & $2.157^{\circ}$ & 0.620 & $\cdot$ \\
\hline Clinidae/Tripterygiidae & $4.090 \cdots$ & $13.328^{\circ}$ & $1.234^{\cdots}$ & 0.159 & $\cdot$ \\
\hline Engraulis australis & $8.209 \cdots$ & $9.752 \mathrm{~ns}$ & $4.877 \cdots$ & 0.394 & ns \\
\hline Gerres subfasciatus & $4.218 \cdots$ & $6.931 \mathrm{~ns}$ & $3.911 \cdots$ & 0.353 & ns \\
\hline Girella tricuspidata & $28.633 \cdots$ & $24.204 \mathrm{~ns}$ & $7.708 \cdots$ & 0.150 & $\cdot$ \\
\hline Gobiidae & $3.656^{\cdots}$ & $5.537 \mathrm{~ns}$ & $2.184^{\cdots}$ & 0.213 & ns \\
\hline Kyphosus sp. & $15.047^{\cdots}$ & $4.211 \mathrm{~ns}$ & $2.228 \cdots$ & 0.281 & ns \\
\hline Labridae & $9.804 \cdots$ & $10.680 \mathrm{~ns}$ & $2.989 \cdots$ & 0.222 & $\cdot$ \\
\hline Mullidae & $0.852 \cdots$ & $9.518^{\circ}$ & $0.593^{\cdots}$ & 0.085 & ns \\
\hline Omobranchus anolius & $0.880 \mathrm{~ns}$ & $1.379 \mathrm{~ns}$ & $0.747 \mathrm{~ns}$ & 0.347 & ns \\
\hline Pagrus auratus & $0.701^{\circ}$ & $0.385 \mathrm{~ns}$ & $0.826^{*}$ & 0.157 & ns \\
\hline Pelates quadrilineatus & $0.423 \mathrm{~ns}$ & $0.880 \mathrm{~ns}$ & $0.574 \mathrm{~ns}$ & 0.452 & $\cdot$ \\
\hline Platycephalidae & $0.829^{\circ}$ & $1.006 \mathrm{~ns}$ & $1.485^{\cdots}$ & 0.139 & $\cdot$ \\
\hline Pomacentridae & $1.311^{*}$ & $1.239 \mathrm{~ns}$ & $0.606^{\circ}$ & 0.179 & - \\
\hline Sardinops neopilchardus & $7.835 \cdots$ & $6.142 \mathrm{~ns}$ & $4.045 \cdots$ & 0.565 & • \\
\hline Scorpididae & $2.023^{\cdots}$ & $1.412 \mathrm{~ns}$ & $1.412 \cdots$ & 0.302 & $\cdot$ \\
\hline Sillago ciliata & $4.785 \cdots$ & $5.808 \mathrm{~ns}$ & $1.823^{\cdots}$ & 0.232 & $\cdot$ \\
\hline
\end{tabular}

Table 4. Summary of ANOVAs of numbers of total taxa, total individuals and individual taxa caught at 2 locations in plume, front and ocean water at North Head, Bondi and Potter Point sewage outfalls in January 1991. Mean square values are shown. Data transformed to $\ln (x+1)$. Other details as in Table 3

\begin{tabular}{|c|c|c|c|c|c|c|c|}
\hline \multirow[t]{3}{*}{ Taxon } & \multicolumn{6}{|c|}{ Source of variation } & \multirow[t]{3}{*}{$\mathrm{CT}$} \\
\hline & Outfall & Location $(\mathrm{O})$ & Water mass & $O \times W$ & $L(O) \times W$ & Residual & \\
\hline & \multicolumn{6}{|c|}{ Degrees of freedom in $F$-test } & \\
\hline Total taxa & $0.037 \mathrm{~ns}$ & $1.212 \cdots$ & $5.784 \mathrm{~ns}$ & $2.345^{\circ}$ & $0.367^{\circ}$ & 0.131 & ns \\
\hline Total individuals & $0.666 \mathrm{~ns}$ & $4.742 \cdots$ & $19.689 \mathrm{~ns}$ & $14.155 \cdots$ & $0.224 n s$ & 0.507 & ns \\
\hline Acanthopagrus australis & $28.693 \mathrm{~ns}$ & $7.298^{\cdots}$ & $18.222 \mathrm{~ns}$ & $6.571 \mathrm{~ns}$ & $2.262 \cdots$ & 0.370 & • \\
\hline Ambassis jacksoniensis & $0.281 \mathrm{~ns}$ & $0.708^{\circ}$ & $2.692 \cdots$ & $0.139 \mathrm{~ns}$ & $0.925 \cdots$ & 0.170 & $\cdot$ \\
\hline Atherinidae & $0.691 \mathrm{~ns}$ & $1.702 \mathrm{~ns}$ & $5.231^{\circ}$ & $0.485 \mathrm{~ns}$ & $0.739 \mathrm{~ns}$ & 0.688 & nis \\
\hline Engraulis australis & $1.868 \mathrm{~ns}$ & $3.772 \cdots$ & $5.187 \mathrm{~ns}$ & $2.732 \mathrm{~ns}$ & $2.085^{\cdots}$ & 0.538 & ns \\
\hline Gerres subfasciatus & $15.278 \mathrm{~ns}$ & $5.032 \cdots$ & $7.187 \mathrm{~ns}$ & $4.965 \mathrm{~ns}$ & $1.714^{\circ}$ & 0.389 & ns \\
\hline Girella tricuspidata & $0.811 \mathrm{~ns}$ & $4.178^{\cdots}$ & $1.727 \mathrm{~ns}$ & $2.540 \mathrm{~ns}$ & $2.431 \cdots$ & 0.388 & ns \\
\hline Gobijdae & $3.810 \mathrm{~ns}$ & $0.812^{\circ}$ & $8.061^{\circ}$ & $0.653 \mathrm{~ns}$ & $0.938^{\cdots}$ & 0.260 & $\cdot$ \\
\hline Gonorynchus greyi & $19.790^{\circ}$ & $0.289 \mathrm{~ns}$ & $4.849 \mathrm{~ns}$ & $4.849^{\cdots}$ & $0.139 \mathrm{~ns}$ & 0.210 & $\cdot$ \\
\hline Kyphosus sp. & $7.883 \mathrm{~ns}$ & $5.233^{\cdots}$ & $8.161 \mathrm{~ns}$ & $5.778 \mathrm{~ns}$ & $1.585^{*}$ & 0.367 & - \\
\hline Labridae & $8.755^{\circ}$ & $0.564^{\circ}$ & $3.407 \mathrm{~ns}$ & $3.459 \mathrm{~ns}$ & $1.669^{\cdots}$ & 0.186 & . \\
\hline Liza argentea & $1.354^{\bullet}$ & $0.097 \mathrm{~ns}$ & $0.173 \mathrm{~ns}$ & $0.998 \mathrm{~ns}$ & $0.293 \mathrm{~ns}$ & 0.228 & $\cdot$ \\
\hline Mullidae & $3.452 \mathrm{~ns}$ & $1.002 \cdots$ & $1.901 \mathrm{~ns}$ & $2.066^{\circ}$ & $0.343 \mathrm{~ns}$ & 0.160 & $\cdot$ \\
\hline Omobranchus anolius & $0.569 \mathrm{~ns}$ & $0.844^{\cdots}$ & 0.330 ns & $0.748 \mathrm{~ns}$ & $0.438^{\circ}$ & 0.149 & $\cdot$ \\
\hline Pagrus auratus & $1.686^{\circ}$ & $0.133 \mathrm{~ns}$ & $0.986 \mathrm{~ns}$ & $0.397 \mathrm{~ns}$ & $0.762 \cdots$ & 0.158 & ns \\
\hline Pelates quadrilineatus & $3.452 \mathrm{~ns}$ & $3.273^{\cdots}$ & $0.844 \mathrm{~ns}$ & 1.310 ns & $1.072 * \cdots$ & 0.092 & · \\
\hline Petroscirtes lupus & $1.297 \mathrm{~ns}$ & $0.278 \mathrm{~ns}$ & $1.202 \mathrm{~ns}$ & $0.680^{\circ}$ & $0.093 \mathrm{~ns}$ & 0.226 & • \\
\hline Platycephalıdae & $1.514 \mathrm{~ns}$ & $0.776^{\circ}$ & $1.175 \mathrm{~ns}$ & $0.645 \mathrm{~ns}$ & $0.330 \mathrm{~ns}$ & 0.246 & $\mathrm{~ns}$ \\
\hline Pomacentridae & $43.428 \mathrm{~ns}$ & $19.765^{\cdots}$ & $1.386 n \mathrm{~ns}$ & 4.153ns & $9.689 \cdots$ & 0.821 & • \\
\hline Sardinops neopilchardus & $1.187 \mathrm{~ns}$ & $0.391^{\cdots}$ & $1.139 \mathrm{~ns}$ & $1.590^{\circ}$ & $0.343^{\cdots}$ & 0.063 & - \\
\hline Sillago ciliata & $9.705^{\circ}$ & 0.089 ns & $3.445 \mathrm{~ns}$ & $2.918 \mathrm{~ns}$ & $1.547^{\cdots}$ & 0.210 & • \\
\hline Trachurus sp. & $13.226^{\bullet}$ & $0.314 \mathrm{~ns}$ & $11.368 \mathrm{~ns}$ & $5.545 \mathrm{~ns}$ & $2.191 \cdots$ & 0.309 & ns \\
\hline Clinidae/Tripterygiidae & $2.632 \mathrm{~ns}$ & $3.457^{\cdots}$ & $11.552 \mathrm{~ns}$ & $6.632 \mathrm{~ns}$ & $1.677^{\cdots}$ & 0.287 & ns \\
\hline
\end{tabular}


Table 5. Mean (+1 SE) numbers of fish caught in plume, front and ocean water at North Head, Bondi and Potter Point sewage outfalls in December 1990

\begin{tabular}{|c|c|c|c|c|c|c|c|c|c|}
\hline \multirow[t]{2}{*}{ Taxon } & \multicolumn{3}{|c|}{ North Head } & \multicolumn{3}{|c|}{ Bondi } & \multicolumn{3}{|c|}{-Potter Point } \\
\hline & Plume & Front & Ocean & Plume & Front & Ocean & Plume & Front & Ocean \\
\hline Acanthopagrus australis & $38.2(9.5)$ & $35.7(1.0)$ & $00(0.0)$ & $43.9(6.4)$ & $249.9(141.6)$ & $0.0(0.0)$ & $0.0(0.0)$ & $0.3(0.3)$ & $0.0(0.0)$ \\
\hline A.therinidae & $0.0(0.0)$ & $25.8(15.8)$ & $0.0(0.0)$ & $0.0(0.0)$ & $13.4(4.1)$ & $0.0(0.0)$ & $1.0(1.0)$ & $0.3(0.3)$ & $0.0(0.0)$ \\
\hline Engraulis australis & $6.3(2.1)$ & $8.8(2.4)$ & $0.0(0.0)$ & $16.3(8.3)$ & $116.8(48.9)$ & $0.0(0.0)$ & $0.3(0.3)$ & $0.6(0.6)$ & $1.6[0.9]$ \\
\hline Gerres subfasciatus & $6.6(1.8)$ & $75.0\{8.9\}$ & $4.2(1.2)$ & $7.6(1.1)$ & $32.0(10.5)$ & $09(0.9)$ & $9.2(3.8)$ & $0.9(0.0)$ & $2.5(1.3)$ \\
\hline Girella tricuspidata & $48.7(2.8)$ & $49.2(12.3)$ & $0.9(0.5)$ & $49.3(7.4)$ & $591.5(325.1)$ & $0.0(0.0)$ & $0.0(0.0)$ & $0.0(0.0)$ & $0.0(0.0)$ \\
\hline Ambassis jacksoniensis & $1.1(1.1)$ & $0.0(0.0)$ & $0.0(0.0)$ & $0.9(0.9)$ & $0.0(0.0)$ & $0.0(0.0)$ & $3.4(0.8)$ & $0.6(0.3)$ & $0.9[0.1]$ \\
\hline Gobiidae & $5.1(2.3)$ & $3.0(1.0)$ & $0.0(0.0)$ & $11.9(2.0)$ & $18.7(9.3)$ & $0.0(0.0)$ & $1.6(0.3)$ & $0.0(0.0)$ & $0.9(0.5)$ \\
\hline Kyphosus sp. & $14.2(5.5)$ & $19.9(6.8)$ & $8.1(2.9)$ & $7.9(1.2)$ & $31.4(12.0)$ & $0.0(0.0)$ & $0.0(0.0\}$ & $0.0(00)$ & $0.0(0.0)$ \\
\hline Labridae & $4.3(0.5)$ & $13.4(3.9)$ & $0.0(0.0)$ & $9.0(3.2)$ & $72.1(36.0)$ & $0.0[0.0)$ & $0.0(0.0)$ & $0.0(0.0)$ & $0.0(0.0)$ \\
\hline Mullidae & $0.0(0.0)$ & $11.5(1.5)$ & $0.9(0.6)$ & $0.0(0.0)$ & $8.0(1.8)$ & $0.0(0.0)$ & $0.0(0.0)$ & $1.9(0.6)$ & $0.3(0.3)$ \\
\hline Omobranchus anolius & $3.9(2.1)$ & $1.5(1.0)$ & $0.0(0.0)$ & $1.3(0.7)$ & $5.2(2.6)$ & $0.4(0.4)$ & $0.7(0.7)$ & $0.3(0.3)$ & $0.3(0.3)$ \\
\hline Pagrus auratus & $0.7(0.7)$ & $0.0(0.0)$ & $0.0(0.0)$ & $0.5(0.5)$ & $4.1(1.6)$ & $0.0(0.0)$ & $0.0(0.0)$ & $0.0(0.0)$ & $0.4(0.4)$ \\
\hline Pelates sexlineatus & $0.8(0.4)$ & $2.2(0.6)$ & $0.0(0.0)$ & $0.4(0.4)$ & $7.7(7.7)$ & $0.0\{0.0\}$ & $0.0(0.0)$ & $0.0(0.0)$ & $0.6(0.3)$ \\
\hline Platycephalidae & $2.0(0.4)$ & $0.4(0.4)$ & $0.0(0.0)$ & $0.0(0.0)$ & $6.5(3.1)$ & $0.0(0.0)$ & $0.0(0.0)$ & $0.0(0.0)$ & $0.0(0.0)$ \\
\hline Pomacentridae & $0.0(0.0)$ & $0.4(0.4)$ & $0.0(0.0)$ & $0.9(0.5)$ & $6.5(3.5)$ & $0.0(0.0)$ & $0.3(0.3)$ & $0.3[0.3]$ & $0.0(0.0)$ \\
\hline Sardinops neopilchardus & $5.8(3.0)$ & $3.6(2.2)$ & $0.6(0.6)$ & $3.6(1.0)$ & $102.8(47.2)$ & $0.0(0.0)$ & $0.0(0.0)$ & $0.0(0.0)$ & $0.0\{0.0\}$ \\
\hline Scorpididae & $0.0(0.0)$ & 0.010 .01 & $0.0(0.0)$ & $0.4(0.4)$ & $17.8(14.2)$ & $0.0(0.0)$ & $0.0(0.0)$ & $0.0(0.0)$ & $0.0(0.0)$ \\
\hline Sillago ciliata & $7.3(0.4)$ & $3.4(1.2)$ & $0.0(0.0)$ & $5.7(2.3)$ & $19.9(8.9)$ & $0.0(0.0)$ & $0.5(0.5)$ & $0.0(0.0)$ & $0.0(0.0)$ \\
\hline Clinidae/Tripterygiidae & $8.0(0.9)$ & $24.7(4.8)$ & $0.0(0.0)$ & $9.3(1.6)$ & $18.8(6.2)$ & $0.0(0.0)$ & $2.2(1.5)$ & $1.4(0.2)$ & $0.0(0.0)$ \\
\hline
\end{tabular}

\section{Developmental stages of young fishes}

Most fish caught were preflexion (Table 1, Figs. $6 \&$ 7). In both periods greatest numbers of pre- and postflexion fish were caught in plumes and fronts at North Head and Bondi, but there were no clear patterns at Potter Point (Figs. 6 \& 7, Table 9) In January the water mass with the greatest numbers of pre-and postflexion fish differed between locations at each outfall (Fig. 7, Table 9). For example, at North Head most preflexion fish were caught in the plume at Location 1, but at the front at Location 2, whereas

Table 6. Mean (+1 SE) numbers of fish caught in plume, front and ocean water at the 2 locations at North Head sewage outfall in January 1991

\begin{tabular}{|c|c|c|c|c|c|c|}
\hline \multirow{2}{*}{ Taxon } & \multicolumn{3}{|c|}{ - Location 1} & \multicolumn{3}{|c|}{ - Location 2} \\
\hline & Plume & Front & Ocean & Plume & Front & Ocean \\
\hline Acanthopagrus australss & $19.7(4.1)$ & $46.7(7.3)$ & $1.7(1.7)$ & $76.7(31.7)$ & $110.7(17.6)$ & $0.3(0.3)$ \\
\hline Ambassis jacksoniensis & $2.7(0.3)$ & $0.0(0.0)$ & $0.0(0.0)$ & $0.0(0.0)$ & $0.0(0.0)$ & $0.0(0.0)$ \\
\hline Atherinidae & $1.0(1.0)$ & $14.7(12.7)$ & $0.0(0.0)$ & $0.3(0.3)$ & $2.7(2.2)$ & $0.0(0.0)$ \\
\hline Engraulis australis & $41.7(12.3)$ & $9.0(2.5)$ & $0.0(0.0)$ & $0.7(0.3)$ & $6.7(4.8)$ & $0.0(0.0)$ \\
\hline Gerres subfasciatus & $0.0(0.0)$ & $2.3(1.3)$ & $0.3(0.3)$ & $1.3(0.9)$ & $39.3(1.3)$ & $0.3(0.3)$ \\
\hline Girella tricuspidata & $0.4(0.4)$ & $3.1(1.7)$ & $0.0(0.0)$ & $5.0(2.5)$ & $9.9(4.6)$ & $1.7(1.2)$ \\
\hline Gobiidae & $9.2(1.5\}$ & $3.9(1.4)$ & $0.0(0.0)$ & $1.7(0.8)$ & $3.3(0.4)$ & $0.0(0.0)$ \\
\hline Gonorynchus greyi & $0.0(0.0)$ & $0.0(0.0)$ & $0.0(0.0)$ & $0.0(0.0)$ & $0.0(0.0)$ & $0.0(0.0)$ \\
\hline Kyphosus sp. & $0.0(0.0)$ & $2.6(1.3)$ & $0.0(0.0)$ & $2.9(1.0)$ & $37.6(4.2)$ & $0.9(0.9)$ \\
\hline Labridae & $18.2(11.2)$ & $8.7(5.1)$ & $0.0(0.0)$ & $0.8(0.8)$ & $22.6(5.0)$ & $0.0(0.0)$ \\
\hline Liza argentea & $0.4(0.4)$ & $0.0\{0.0\}$ & $0.0(0.0)$ & $0.9(0.4)$ & $0.0(0.0)$ & $0.0(0.0)$ \\
\hline Mullıdae & $0.0(0.0)$ & $0.5(0.5)$ & $0.0(0.0)$ & $0.0(0.0)$ & $0.8(0.8)$ & $0.0(0.0)$ \\
\hline Omobranchus anolus & $0.4(0.4)$ & $0.0(0.0)$ & $0.0(0.0)$ & $0.0(0.0)$ & $1.9(0.4)$ & $0.0(0.0)$ \\
\hline Pagrus auratus & $0.4(0.4)$ & $1.7(0.4)$ & $0.4(0.4)$ & $6.3(1.3)$ & $0.8(0.8)$ & $0.0(0.0)$ \\
\hline Pelates sexlineatus & $0.0(0.0)$ & $0.5(0.5)$ & $0.0(0.0)$ & $0.0(0.0)$ & $0.0(0.0)$ & $0.0(0.0)$ \\
\hline Petroscurtes lupus & $0.0(0.0)$ & $0.0(0.0)$ & $0.0(0.0)$ & $0.0(0.0)$ & $1.2(0.7)$ & $0.0(0.0)$ \\
\hline Platycephalidae & $1.3(0.7)$ & $0.0(0.0)$ & $0.5(0.5)$ & $0.4\{0.4\}$ & $0.4(0.4)$ & $0.0(0.0)$ \\
\hline Pomacentridae & $3.4(1.5)$ & $0.4(0.4)$ & $0.0\{0.0\}$ & $0.0(0.0)$ & $1.0(0.6)$ & $0.0(0.0)$ \\
\hline Sardinops neopilchardus & $0.0(0.0)$ & $8.1(1.8)$ & $0.0(0.0)$ & $0.0(0.0)$ & $1.4(0.7)$ & $0.0(0.0)$ \\
\hline Sillago ciliata & $30.2(17.6)$ & $3.0(1.8)$ & $0.0(0.0)$ & $3.3(0.7)$ & $15.9(5.4)$ & $0.0(0.0)$ \\
\hline Trachurus sp. & $39.7(17.9)$ & $5.3(3.4)$ & $0.0(0.0)$ & $38.0(5.2)$ & $11.5(6.9)$ & $0.4(0.4)$ \\
\hline Clinidae/Tripterygiıdae & $2.9(1.5)$ & $20 \quad(5.9)$ & $0.0(0.0)$ & $0.0(0.0)$ & $41.8(14.4)$ & $0.0(0.0)$ \\
\hline
\end{tabular}


Table 7 Mean ( +1 SE) numbers of fish caught in plume, front and ocean water at the 2 locations at Bondi sewage outfall in January 1991

\begin{tabular}{|c|c|c|c|c|c|c|}
\hline \multirow{2}{*}{ Taxon } & \multicolumn{3}{|c|}{ Location $1 \longrightarrow$} & \multirow[b]{2}{*}{ Plume } & \multirow{2}{*}{-Location 2- } & \multirow[b]{2}{*}{ Ocean } \\
\hline & Plume & Front & Ocean & & & \\
\hline Acanthopagrus australis & $33.3(19.0)$ & $72.7(61.2)$ & $0.0(0.0)$ & $0.3(0.3)$ & $0.0(0.0)$ & $0.0(0.0)$ \\
\hline Ambassis jacksoniensis & $3.0(2.5)$ & $1.7(1.2)$ & $0.0(0.0)$ & $0.3(0.3)$ & $0.0(0.0)$ & $0.0\{0.0\}$ \\
\hline Atherinidae & $2.3(2.3)$ & $20.3(15.5)$ & $0.0(0.0)$ & $0.0(0.0)$ & $0.7(0.7)$ & $0.0(0.0)$ \\
\hline Engraulis australis & $3.3(0.9)$ & $11.3(8.4)$ & $0.0(0.0)$ & $0.3(0.3)$ & $1.0(1.0)$ & $0.0(0.0)$ \\
\hline Gerres subfasciatus & $44.0(28.0)$ & $49.0(17.6)$ & $2.0(1.2)$ & $11.7(5.2)$ & $3.3(3.3)$ & $1.0(1.0)$ \\
\hline Girella tricuspidata & $2.1(0.4)$ & $9.4(3.5)$ & $0.0(0.0)$ & $0.0(0.0)$ & $1.1(1.1)$ & $0.0(0.0)$ \\
\hline Gobiidae & $2.1(0.4)$ & $1.3(0.8)$ & $0.0(0.0)$ & $0.5(0.5)$ & $0.0(0.0)$ & $0.0(0.0)$ \\
\hline Gonorynchus greyi & $3.2(1.3)$ & $39.4(16.5)$ & $2.4(0.3)$ & $1.4(0.8)$ & $63.3(29.2)$ & $0.7(0.7)$ \\
\hline Kyphosus sp. & $22.4(5.6)$ & $38.9(15.7)$ & $0.0(0.0)$ & $2.4(0.5)$ & $14.4(11.0)$ & $0.3(0.3)$ \\
\hline Labridae & $0.4(0.4)$ & $0.0(0.0)$ & $0.0(0.0)$ & $0.0(0.0)$ & $0.0(0.0)$ & $0.0(0.0)$ \\
\hline Liza argentea & $0.9(0.4)$ & $4.5(2.7)$ & $0.0(0.0)$ & $0.0(0.0)$ & $3.2(2.4)$ & $1.2(0.7)$ \\
\hline Mullidae & $0.4(0.4)$ & $15.1\{8.6\}$ & $2.0(0.9)$ & $0.5(0.5)$ & $3.7(0.7)$ & $0.0(0.0)$ \\
\hline Omobranchus anolius & $4.8(3.0)$ & $2.2(1.6)$ & $0.0(0.0)$ & $0.5(0.5)$ & $0.0(0.0)$ & $0.0(0.0)$ \\
\hline Pagrus auratus & $0.0(0.0)$ & $0.4(0.4)$ & $0.0(0.0)$ & $0.0(0.0)$ & $0.0(0.0)$ & $0.0(0.0)$ \\
\hline Pelates sexlineatus & $5.5(1.2)$ & $20.5(8.9)$ & $0.0(0.0)$ & $0.5(0.5)$ & $0.0(0.0)$ & $0.0(0.0)$ \\
\hline Petroscirtes lupus & $0.8(0.8)$ & $4.9(2.5)$ & $0.6(0.6)$ & $0.0(0.0)$ & $3.1(1.9)$ & $0.0(0.0)$ \\
\hline Platycephalidae & $0.0(0.0)$ & $0.9(0.4)$ & $0.0(0.0)$ & $0.0(0.0)$ & $0.0(0.0\}$ & $0.0(0.0)$ \\
\hline Pomacentridae & $0.0(0.0)$ & $1.4(1.4)$ & $0.0(0.0)$ & $0.0(0.0)$ & $0.0(0.0)$ & $0.0(0.0)$ \\
\hline Sardnops neopilchardus & $0.0(0.0)$ & $0.0(0.0)$ & $0.0(0.0)$ & $0.0(0.0)$ & $0.0(0.0)$ & $0.0(0.0)$ \\
\hline Sillago ciliata & $0.0(0.0)$ & $2.2(1.2)$ & $0.0(0.0)$ & $0.5(0.5)$ & $0.0(0.0)$ & $0.0(0.0)$ \\
\hline Trachurus sp. & $0.4(0.4)$ & $0.4(0.4)$ & $0.6(0.6)$ & $0.0(0.0)$ & $0.0(0.0)$ & $0.0(0.0)$ \\
\hline Clinidae/Tripterygindae & $6.6(2.8)$ & $11.2(5.4)$ & $0.0(0.0)$ & $0.0(0.0)$ & $0.5(0.5)$ & $0.0(0.0)$ \\
\hline
\end{tabular}

at Potter Point most preflexion fish occurred in the ocean at Location 1, but there was no significant difference between plume and ocean water at Location 2 (Fig. 7).
A greater percentage of preflexion fish were caught in all water masses at North Head and Bondi in December, but this was not the case at Potter Point (Fig. 6). In January a greater percentage of preflexion

Table 8. Mean ( $+1 \mathrm{SE})$ numbers of fish caught in plume, front and ocean water at the 2 locations at Potter Point sewage outfall in January 1991

\begin{tabular}{|c|c|c|c|c|c|c|}
\hline \multirow{2}{*}{ Taxon } & \multicolumn{3}{|c|}{ Location 1} & \multicolumn{3}{|c|}{-Location 2} \\
\hline & Plume & Front & Ocean & Plume & Front & Ocean \\
\hline Acanthopagrus australis & $1.3(0.9)$ & $0.7(0.7)$ & $0.0(0.0)$ & $0.0(0.0)$ & $0.0(0.0)$ & $0.7(0.3)$ \\
\hline Ambassis jacksoniensis & $4.3(0.9)$ & $0.0(0.0)$ & $0.0(0.0)$ & $0.7(0.7)$ & $1.3(0.9)$ & $0.0(0.0)$ \\
\hline Atherinidae & $1.0\{1.0\}$ & $4.3(4.3)$ & $0.0(0.0)$ & $0.0\{0.0\}$ & $0.0(0.0)$ & $0.0(0.0)$ \\
\hline Engraulis australis & $2.3(1.2)$ & $3.0(1.7)$ & $1.0(0.6)$ & $1.7(0.9)$ & $0.0(0.0)$ & $4.7(2.4)$ \\
\hline Gerres subfasclatus & $0.0(0.0)$ & $0.7(0.7)$ & $0.3(0.3)$ & $0.0(0.0)$ & $0.0(0.0)$ & $0.0(0.0)$ \\
\hline Girella tricuspidatá & $0.0(0.0)$ & $4.5(2.6)$ & $0.5(0.5)$ & $3.2(0.9)$ & $0.0(0.0)$ & $22.3(10.9)$ \\
\hline Gobridae & $9.8(1.7)$ & $4.0(4.0)$ & $1.5(0.9)$ & $4.2(0.5)$ & $5.7(1.5)$ & $0.0(0.0)$ \\
\hline Gonorynchus greyi & $0.0(0.0)$ & $0.0(0.0)$ & $0.0(0.0)$ & $0.0(0.0)$ & $0.0(0.0)$ & $0.0(0.0)$ \\
\hline Kyphosus sp. & $0.5(0.5)$ & $0.0(0.0)$ & $0.0(0.0)$ & $0.5(0.5)$ & $0.0(0.0)$ & $3.4(1.0)$ \\
\hline Labridae & $2.4(0.5)$ & $0.0(0.0)$ & $0.4(0.4)$ & $0.0(0.0)$ & $0.0(0.0)$ & $0.0(0.0)$ \\
\hline Liza argentea & $0.0(0.0)$ & $0.0(0.0)$ & $0.0\{0.0\}$ & $0.0(0.0)$ & $0.0(0.0)$ & $1.2(0.7)$ \\
\hline Mullidae & $0.0(0.0)$ & $0.0(0.0)$ & $0.0(0.0)$ & $0.5(0.5)$ & $0.0(0.0)$ & $2.3(0.6)$ \\
\hline Omobranchus anolius & $0.0(0.0)$ & $0.0(0.0)$ & $0.5(0.5)$ & $0.0(0.0)$ & $0.0(0.0)$ & $0.5(0.5)$ \\
\hline Pagrus auratus & $0.5(0.5)$ & $1.0(1.0)$ & $0.0(0.0)$ & $0.6(0.6)$ & $0.5(0.5)$ & $0.0(0.0)$ \\
\hline Pelates sexineatus & $0.0(0.0)$ & $0.0(0.0)$ & $0.0(0.0)$ & $0.0(0.0)$ & $0.0(0.0)$ & $0.9(0.4)$ \\
\hline Petroscirtes lupus & $0.5(0.5)$ & $0.0(0.0)$ & $0.0(0.0)$ & $0.0(0.0)$ & $0.0(0.0)$ & $0.0(0.0)$ \\
\hline Platycephalidae & $5.9(2.2)$ & $3.5(1.8)$ & $0.0(0.0)$ & $1.1(1.1)$ & $1.0(1.0)$ & $0.0(0.0)$ \\
\hline Pomacentridae & $5.9(0.0)$ & $23.9(13.3)$ & $121.8(16.7)$ & $21.9(17.2)$ & $0.0(0.0)$ & $0.5(0.5)$ \\
\hline Sardinops neopilchardus & $0.0(0.0)$ & $0.0(0.0)$ & $0.0(0.0)$ & $0.5(0.5)$ & $0.0(0.0)$ & $0.5(0.5)$ \\
\hline Sillago ciliata & $0.0(0.0)$ & $0.0(0.0)$ & $0.0(0.0)$ & $0.0(0.0)$ & $0.0(0.0)$ & $0.4(0.4)$ \\
\hline Trachurus sp. & $24.6(5.9)$ & $0.0(0.0)$ & $0.0(0.0)$ & $1.6(0.9)$ & $1.6(1.6)$ & $4.6(1.9)$ \\
\hline Clinidae/Tripterygiidae & $12.8(2.8)$ & $1.0(1.0)$ & $0.0(0.0)$ & $0.5(0.5)$ & $0.0(0.0)$ & $0.0(0.0)$ \\
\hline
\end{tabular}



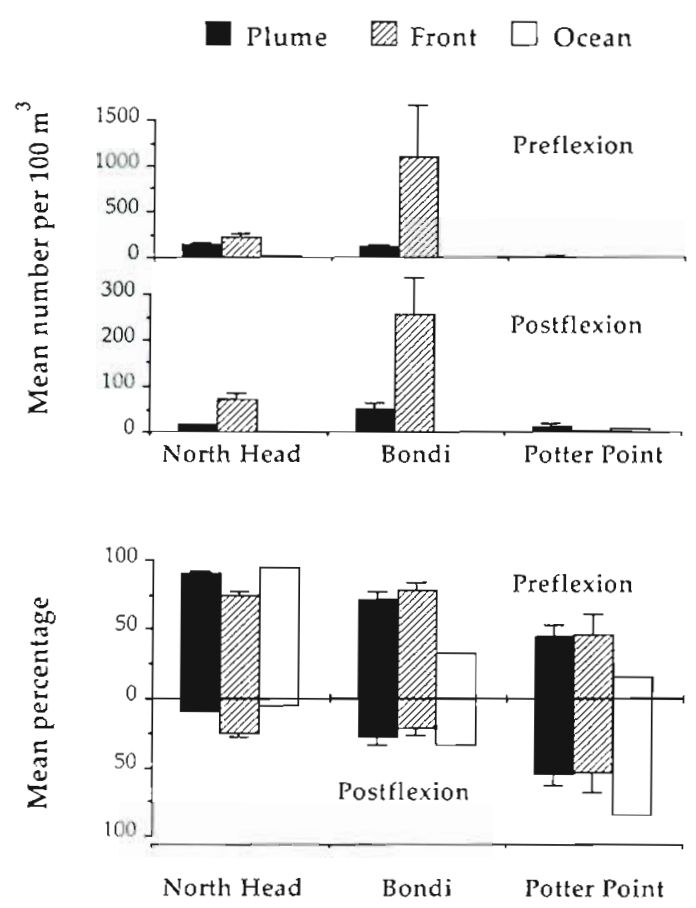

Fig. 6. Mean (+1 SE) numbers and proportions of pre- and postflexion fishes caught in plume, front and ocean waters at the North Head, Bondi and Potter Point sewage outfalls in December 1990
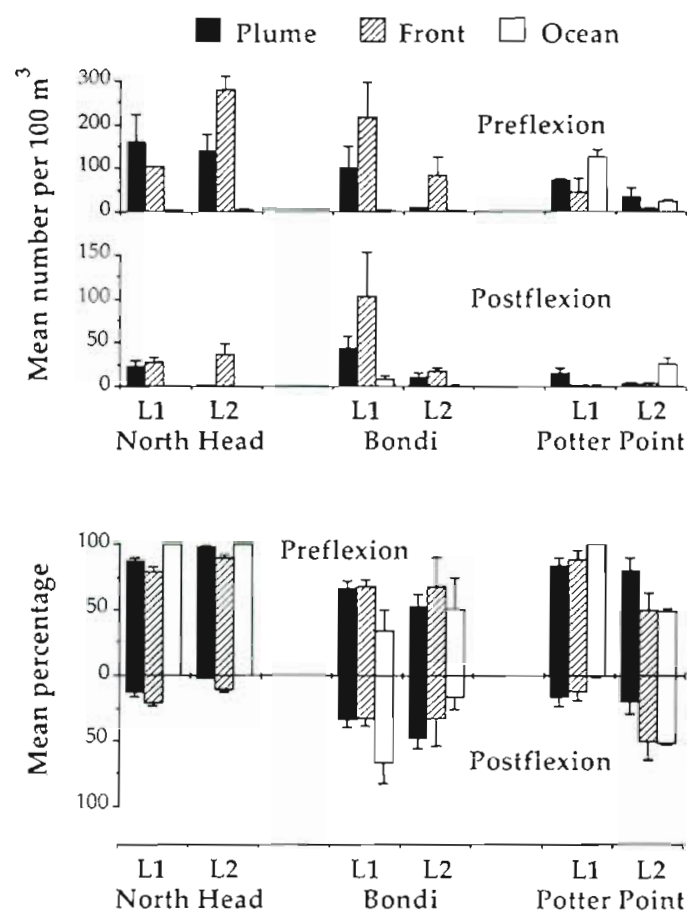

Fig. 7 Mean (+1 SE) numbers and proportions of preand postflexion fishes caught in plume, front and ocean waters at the North Head, Bondi and Potter Point sewage outfalls in January 1991 L1 = Location 1; L2 = Location 2 fish was caught in al] water masses at both locations at North Head, but there were no consistent trends at Bondi and Potter Point (Fig. 7, Table 9).

Postflexion fishes, including some well developed juveniles of several taxa, notably Sardinops neopilchardus, Engraulis australis, Atherinidae, Hemiramphidae, Stromateidae, Mullidae, Teraponidae and Blenniidae, were caught at fronts (Table 1).

\section{Flotsam and drift algae}

Small amounts of flotsam, including drift algae and seagrass shoots, and garbage, such as plastic bags and containers, were collected at fronts of all 3 plumes. Greatest amounts of drift algae/seagrass were collected at fronts at North Head (Table 10). There were no significant relationships between the quantity of drift algae collected and total numbers of fish caught at fronts (Fig. 8). Mulched toilet paper was collected in all plume samples.
Table 9. Summary of ANOVAs of numbers and percentages of pre-and postflexion fishes caught in plume, front and ocean water at North Head, Bondi and Potter Point sewage outfalls in December 1990 and January 1991. Abundance data transformed to $\ln (x+1)$, percentage data transformed to arcsin. df: degrees of freedom; MS: mean square. Other symbols as in Tables $3 \& 4$

\begin{tabular}{|c|c|c|c|c|c|}
\hline & \multirow[t]{3}{*}{$\mathrm{df}$} & \multirow{2}{*}{\multicolumn{2}{|c|}{$\begin{array}{c}\text { Abundance } \\
\text { Preflexion Postflexion }\end{array}$}} & \multirow{2}{*}{\multicolumn{2}{|c|}{$\begin{array}{c}\text { Percentage } \\
\text { Preflexion Postflexion }\end{array}$}} \\
\hline & & & & & \\
\hline & & MS & MS & MS & MS \\
\hline \multicolumn{6}{|l|}{ December 1990} \\
\hline Outfall & 2 & $23.783 \cdots$ & $3.605 \cdots$ & $1.164^{\cdots}$ & $0.929^{\circ}$ \\
\hline Water mass & 2 & $28.016 \mathrm{~ns}$ & $15.533 \mathrm{~ns}$ & $0.047 \mathrm{~ns}$ & $0.159 \mathrm{~ns}$ \\
\hline$O \times W$ & 4 & $5.930^{\cdots}$ & $6.513 \cdots$ & $0.169 \mathrm{~ns}$ & $0.118 \mathrm{~ns}$ \\
\hline Residual & 18 & 0268 & 0.320 & 0.122 & 0.132 \\
\hline Total & 26 & & & & \\
\hline Cochran's test & & ns & ns & ns & ns \\
\hline \multicolumn{6}{|l|}{ January 1991} \\
\hline Outfall & 2 & $3.103 \mathrm{~ns}$ & $4.076 \mathrm{~ns}$ & $1.836 \mathrm{~ns}$ & 0.501 ns \\
\hline Location $(\mathrm{O})$ & 3 & $5.503 \cdots$ & $4.917 \cdots$ & $0.493^{\cdots}$ & $0.199 \cdots$ \\
\hline Water mass & 2 & $20.221 \mathrm{~ns}$ & $15.993 \mathrm{~ns}$ & $0.088 \mathrm{~ns}$ & $0.011 \mathrm{~ns}$ \\
\hline $\mathrm{O} \times \mathrm{W}$ & 4 & $13.952 \cdots$ & $5.923 \mathrm{~ns}$ & $0.300 \mathrm{~ns}$ & $0.040 \mathrm{~ns}$ \\
\hline$L(O) \times W$ & 6 & $0.609 \mathrm{~ns}$ & $2.650^{\cdots}$ & $0.148^{\circ}$ & $0.116^{\cdots}$ \\
\hline Residual & 36 & 0.624 & 0.263 & 0.053 & 0.029 \\
\hline Total & 53 & & & & \\
\hline Cochran's test & & ns & ns & ns & ns \\
\hline
\end{tabular}


Table 10. Mean weight $(\mathrm{g})(+1 \mathrm{SE})$ of drift algae/seagrass collected in plume, front and ocean waters at North Head, Bondi and Potter Point sewage outfalls in December 1990 and January 1991

\begin{tabular}{|c|c|c|c|}
\hline & North Head & Bondi & Potter Point \\
\hline \multicolumn{4}{|c|}{ December 1990} \\
\hline Plume & $0 \quad\{0\}$ & $\begin{array}{ll}0 & (0)\end{array}$ & $0 \quad\{0\}$ \\
\hline Front & $17.5(5.6)$ & $8.4(4.4)$ & $0 \quad\{0\}$ \\
\hline Ocean & $1.4(1.4)$ & $0 \quad(0)$ & $0 \quad(0)$ \\
\hline \multicolumn{4}{|c|}{ January 1991} \\
\hline \multicolumn{4}{|c|}{ Location 1} \\
\hline Plume & $0 \quad(0)$ & $\begin{array}{ll}0 & (0)\end{array}$ & $0 \quad(0)$ \\
\hline Front & $20.8(5.4)$ & $0 \quad(0)$ & $0.9(0.9)$ \\
\hline Ocean & $1.3(0.8)$ & $\begin{array}{ll}0 & (0)\end{array}$ & $\begin{array}{ll}0 & (0)\end{array}$ \\
\hline \multicolumn{4}{|c|}{ Location 2} \\
\hline Plume & $0 \quad(0)$ & $\begin{array}{ll}0 & (0)\end{array}$ & $\begin{array}{ll}0 & 10\end{array}$ \\
\hline Front & $10.5(3.9)$ & $\begin{array}{ll}0 & (0)\end{array}$ & $\begin{array}{ll}0 & (0)\end{array}$ \\
\hline Ocean & $0 \quad(0)$ & $0 \quad(0)$ & $\begin{array}{ll}0 & (0)\end{array}$ \\
\hline
\end{tabular}

\section{DISCUSSION}

\section{Oceanography of surface sewage plumes}

Sewage plumes formed significant physical intrusions into mainstream continental shelf waters off Sydney. These plumes can extend many kilometres seaward and alongshore (observed up to $5 \mathrm{~km}$ seaward and $8 \mathrm{~km}$ alongshore; see also Gray 1995, Kingsford \& Gray 1996). This is of similar extent to that of estuarine plumes from Botany Bay (observed up to $11 \mathrm{~km}$ offshore; Kingsford \& Suthers 1994). However, both sewage and estuarine plumes were mostly confined to within $2 \mathrm{~km}$ of the coast, and aligned in alongshore currents. Variations in orientation and spatial extent of sewage plumes over the continental shelf would probably be caused by variations in the amount and rates of discharge among outfalls and throughout time, differing oceanographic conditions, and the degree of mixing of plume and shelf waters. In contrast to estuarine plumes, the oceanographic structure of sewage plumes did not alter significantly with tidal phase. Thus sewage plumes formed persistent intrusions into the shelf waters off Sydney, potentially posing a constant structure that may influence fishes during early development.

The oceanography of each sewage plume was similar and resembled that of estuarine/riverine plumes, i.e. a shallow wedge of turbid lower-salinity water overlaying clear high-salinity shelf water (Govoni et al. 1989, Grimes \& Finucane 1991, Kingsford \& Suthers 1994). The abserved depth of the sewage plumes ranged between 1 and $5 \mathrm{~m}$ and a distinct frontal region (1 to $3 \mathrm{~m}$ wide) was usually associated with each

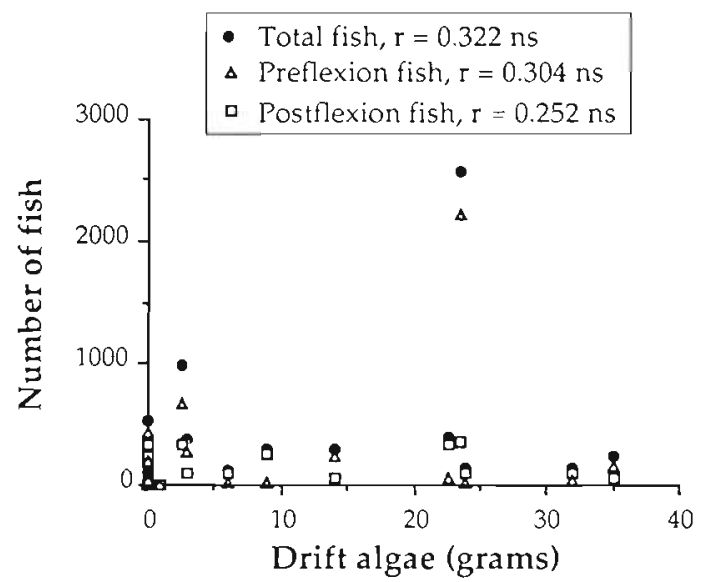

Fig. 8. Relationships between numbers of young fish and weight of drift algae/seagrass collected at the fronts of sewage plumes. Data for all 3 outfalls and both sampling periods combined ( $n=27$ ). The correlation coefficient $(r)$ and its significance are given for each correlation. ns: not significant

plume. This front was observed as a distinct change in turbidity, colour and usually salinity (see also Kingsford \& Gray 1996). The accumulation of drift algae and flotsam along fronts of sewage plumes suggested these fronts were areas of convergence, similar to that described for estuarine plumes (Govoni \& Grimes 1992) and other oceanographic features (Kingsford 1990). The depth of the sewage plumes, the width and intensity of the front and the disparity in salinity change at the front (see Kingsford \& Gray 1996), and thus the intensity of convergence of surface waters around these plumes, would probably be influenced by the rate and quantity of the discharge, distance from the point-source and interactions of plumes with surrounding shelf waters and bottom topography (e.g. Wolanski \& Hamner 1988, Govoni \& Grimes 1992). Thus, oceanographic influences of plumes on young fish and other plankton may vary according to interactions between plume and shelf waters.

\section{Influences of surface sewage plumes on distributions of young fishes}

Surface sewage plumes are capable of influencing small-scale $(<1 \mathrm{~km})$ patterns of distribution of larval and presettlement fishes in coastal waters. Densities of a wide taxonomic suite of fish were usually greater in plumes or fronts than in adjacent shelf water, which concurs with that reported for small fish and plankton in many types of natural oceanographic features (see 'Introduction'). Increased concentrations of small fishes along frontal regions of oceanographic features may be due to a combination of active and passive 
processes, but have often been attributed to oceanographic convergence (e.g. Govoni et al. 1989, Kingsford 1990, Sabates 1990). The similarity in oceanography of sewage plumes to natural plumes suggests that convergence of surface waters may have facilitated the concentration of young fishes along the fronts of sewage plumes. Passive concentration would most likely be the case for preflexion fishes, but postflexion fishes may have been attracted to these plumes by other factors, such as the potential increase in prey concentrations. Increased catches of some small preflexion fish in plumes may have been the result of the fibrous material in plumes altering the functional mesh size and sampling efficacy of the net (see Kingsford \& Gray 1996). However, this does not negate the fact that large numbers of young fish were found in plumes and were thus exposed to pollutants. Future work is required to assess the effects of fibrous materials in plumes on catchability of young fishes.

Aggregation of fishes at fronts was variable. Rank abundances of fishes among water masses varied among taxa, locations around an outfall (e.g. Sillago ciliata and Labridae at North Head in January) and throughout time. This variability concurred with that documented between these sewage plumes and distant shelf waters (Gray et al. 1992). Such variation among water masses was not unexpected given the inherent heterogeneity in distributions of planktonic organisms (Haury et al. 1978, Gray 1993, 1996, Thorrold et al. 1994), changes in behaviour, immigration and emigration of young fishes from plumes, and spatial and temporal variation in the physical attributes of each outfall and plume. In particular, changes in the behaviour and intensity of the front along different parts of the plume would probably be important in concentrating fish, and require greater investigation.

Small quantities of drift algae and flotsam were collected at fronts. Although there were no significant correlations between quantity of drift algae and abundances of fish, these structures may have accounted for higher abundances of some organisms at fronts. In particular, certain types of small fishes (e.g. postflexion Hemiramphidae, Exocoetidae, Carangidae, Stromateidae, Mullidae and Mugilidae) may have been attracted to, or were living among, clumps of drift algae collected in fronts. Small fishes have been documented as being associated with floating objects, such as drift algae, elsewhere (Hunter \& Mitchell 1967. Mitchell \& Hunter 1970, Safran \& Omori 1990, Kingsford 1992, 1993).

Depth of the sewage plumes suggested that greatest influences on planktonic organisms would be in surface waters ( 1 to $3 \mathrm{~m}$ ). However, plumes are 3-dimensional structures (e.g. Wolanski \& Hamner 1988) and may affect vertical patterns of water circulation and vertical movements and transportation of small fish and plankton, particularly those that migrate vertically (e.g. Kendall \& Naplin 1981, Brewer \& Kleppel 1986, Haney 1988, Neilson \& Perry 1990). Subduction at the front may displace young fish to a deeper layer of water (Franks 1992). Moreover, young fish and plankton may be concentrated at the subsurface halocline between plume and ocean, similar to that around subsurface thermoclines (Kendall \& Naplin 1981, Frost 1988, Haney 1988).

Sewage plumes move across and along the continental shelf and may modify the distributions and densities of young fish and other plankton over large spatial scales $(>1 \mathrm{~km})$. The size and orientation of these sewage plumes off Sydney suggest that fish in surface waters within at least $5 \mathrm{~km}$ of the outfalls are likely to encounter these plumes. These plumes could thus alter cross-shelf distributions of young fishes. In particular, fish may be entrained in, or follow, plumes and fronts, potentially altering their patterns of transport and recruitment, similar to other oceanographic processes (Shanks 1983. Grimes \& Finucane 1991). Exposure of young fish to sewage plumes may vary among different taxa, depending on where they were spawned and where they spend their early life, larval behaviour and oceanography. Surface dwelling taxa that predominately occur in nearshore waters during their early development (e.g Tripterygiidae, Acanthopagrus australis, Sillago ciliata) would probably be most vulnerable to sewage plumes. Furthermore, ontogenetic shifts in horizontal and vertical distributions displayed by some taxa (e.g. Liza argentea) may mean that either smaller or larger individuals of a species are affected by these plumes. For example, larger $L$. argentea occur in surface waters (Gray 1993). The oceanographic conditions off Sydney change throughout the year, influencing both the distributions of young fishes and the behaviour of sewage plumes. For example, in winter (July-August) offshore winds predominate, sweeping plumes greater distances offshore, potentially enhancing exposure to a wider suite of taxa. In winter many oceanic pelagic taxa (e.g. Myctophidae) are transported onto the inner shelf off Sydney (Gray 1993, 1995). In winter, therefore, a wider suite of nearshore and offshore taxa may be affected by these plumes.

The oceanography of sewage plumes may enhance and prolong exposure of young fishes to pollutants. Pollutants (e.g. organochlorines) are often concentrated at fronts (Hardy et al. 1985, Brown et al. 1991, Tanabe et al. 1991), similar to the sea-surface microlayer (Hardy 1982, Hardy et al. 1987a), and models that assume simple dilution of pollutants from pointsources are inappropriate (see also Cross et al. 1987 , Hardy et al. 1987b). Entrainment of fish and pollutants in plumes and fronts may create a greater risk to sur- 
vival during early development. Quantifying lethal effects of plumes on young fishes in the field would be extremely difficult given that fish may not necessarily die from contact with plumes immediately, but may die further downstream from the actual outfall. In the absence of toxic substances and lethal effects, the potential increased food resources in sewage plumes could be beneficial to young fish.

The situation of multiple sewage plumes off Sydney ( 7 shoreline outfalls within $50 \mathrm{~km}$ of coastline) may significantly enhance exposure to pollutants and effects of plumes on young fishes. Plumes from the 3 largest shoreline outfalls (North Head, Bondi and Malabar) sometimes merged (author's pers, obs.), potentially creating 1 large polluted field covering virtually all the nearshore waters off Sydney. Reversing currents, eddies and other oceanographic events may also lead to prolonged exposure. At certain times it would be possible for fish to be swept by alongshore currents through all plumes within a couple of hours. These plumes often covered the entrances to the main estuaries in this region; Sydney Harbour, Botany Bay and Port Hacking These plumes, therefore, may not only have affected young fish in coastal waters, but fish entering or leaving these estuaries. Subsequently, these plumes could have considerable influence on the recruitment dynamics of small fishes and other meroplankton in both nearshore and estuarine waters around Sydney. It is plausible that sizes of adult stocks of these organisms around Sydney may be affected due to the loss of young.

Of greater concern is that, unlike benthic organisms and adult fishes, young fishes from a wide field may be affected by sewage plumes, and effects may be manifest over large spatial scales (see also Keough \& Black 1996. Raimondi \& Reed 1996). Young fishes entrained in mainstream coastal water bodies that were spawned tens to hundreds of kilometres upstream may be transported through these plumes, potentially affecting supply of recruits to downstream areas. Small changes in rates of mortality during early development can have dramatic effects on levels of recruitment (Underwood \& Fairweather 1989, Fogarty et al. 1991). Therefore, sewage plumes could influence the dynamics and vitality of marine communities over a wide area of a coast, particularly if many such outfalls occur along a coastline, as in New South Wales (40 ocean sewage outfalls occur along $1000 \mathrm{~km}$ of coastline).

Ultimately, location of the plume point-source, the amount, type and concentration of effluent, the place and timing of spawning, length of the pelagic phase, distribution and behaviour patterns of young fish and their resilience to pollutants, and interactions with oceanography will determine taxa most vulnerable to anthropogenic plumes and potential influences to adult stock. Effects on health and condition of young fishes (review in Ferron \& Leggett 1994) and genetic, immunological and teratological (e.g. Longwell et al. 1992, Bodammer 1993, MacLean 1993) influences of plumes on development of embryos and larvae need to be considered. Both field and laboratory studies may be needed to successfully quantify effects of sewage plumes on young fishes in the future (e.g. Hardy et al. $1987 \mathrm{~b}$ ). Future field studies need to place more emphasis on oceanography in modifying the effects of pollutants on young fishes.

Acknowledgements. J. Craig modified the research vessel and assisted with some sampling. K. Elder gave much support and assisted with some sampling. $M$. Kingsford, N. Otway, A. Miskiewicz and J. Leis provided discussions and comments on the research.

\section{LITERATURE CITED}

Alldredge AL, Hamner WM (1980) Recurring aggregation of zooplankton by a tidal current. Estuar Coast Mar Sci 10: $31-37$

Arfi R, Champalbert G, Patriti G (1981) Système planctonique et pollution urbaine: un aspect des populations zooplanctoniques. Mar Biol 61:133-141

Beder S (1989) Toxic fish and sewer surfing. Allen and Urwin, Sydney

Bodammer JE (1993) The teratological and pathological effects of contaminants on embryonic and larval fishes exposed as embryos: a brief review. Am Fish Soc Symp 14: $77-84$

Brewer GD, Kleppel GS (1986) Diel vertical distnbution of fish larvae and their prey in nearshore waters of southern California. Mar Ecol Prog Ser 27:217-226

Brown J, Turrell WR, Simpson JH (1991) Aerial surveys of axial convergent fronts in UK estuaries and the implications for pollution. Mar Pollut Bull 22:397-400

Clarke KR (1993) Non-parametric multivariate analyses of changes in community structure. Aust J Ecol 18:117-143

Costello MJ, Read P (1994) Toxicity of sewage sludge to marine organisms: a review. Mar Environ Res 37:23-46

Cross JN, Hardy JT, Hose JE, Hershelman GP, Antrim LD, Gossett RW, Crecelius EA (1987) Contaminant concentrations and toxiclty of sea-surface microlayer near Los Angeles, California. Mar Environ Res 23:307-323

Denman KL, Powell TM (1984) Effects of physical processes on planktonic ecosystems in the coastal ocean. Oceanogr Mar Biol A Rev 22:125-168

Duedall IW, Ketchum BH, Park. PK, Kester DR (eds) (1983) Wastes in the ocean, Vol 1, Industrial and sewage wastes in the ocean. Wiley, New York

Emery AR (1972) Eddy formation from an oceanic island: ecological effects. Caribb J Sci 12:121-128

Epifanio CE (1987) The role of tidal fronts in maintaining patches of Brachyuran zoeae in estuarne waters. J Crust Biol 7:513-517

Fagan P, Miskiewicz AG, Tate PM (1992) An approach to monitoring sewage outfalls: a case study on the Sydney deepwater sewage outfalls. Mar Pollut Bull 25:172-180

Ferron A, Leggett WC (1994) An apprassal of condition measures for marine fish larvae. Adv Mar Biol 30: $217-303$ 
Field JG, Clarke KR, Warwick RM (1982) A practical strategy for analysing multispecies distribution patterns. Mar Ecol Prog Ser 8:37-52

Fogarty MJ, Sissenwine MP, Cohen EB (1991) Recruitment variability and the dynamics of exploited marine populations. Trends Ecol Evol 6:241-246

Franks PJS (1992) Sink or swim: accumulation of biomass at fronts. Mar Ecol Prog Ser 82:1-12

Frost BW (1988) Variability and possible adaptive significance of diel vertical migration in Calanus pacificus a planktonic marine copepod. Bull Mar Sci 43:675-694

Govoni JJ (1993) Flux of larval fishes across frontal boundaries: examples from the Mississippi River plume front and the western Gulf Stream front in winter Bull Mar Sci 53: $538-566$

Govoni JJ, Grimes CB (1992) The surface accumulation of larval fishes by hydrodynamic convergence within the Mississippi River plume front. Cont Shelf Res 12: $1265-1276$

Govoni JJ, Hoss DE, Colby DR (1989) The spatial distribution of larval fishes about the Mississippi River Plume. Limnol Oceanogr 34:178-187

Gray CA (1993) Horizontal and vertical trends in the distribution of larval fishes in coastal waters off central New South Wales, Australia. Mar Biol 1 16:649-666

Gray CA (1995) The influences of sewage plumes and oceanography on assemblages of larval fishes. PhD thesis, Univ Sydney

Gray CA (1996) Small-scale temporal variability in assemblages of larval fishes: implications for sampling. J Plankton Res 18:in press

Gray CA, Otway NM, Laurenson FA, Miskıewicz AG, Pethebridge RL (1992) Distribution and abundance of marine fish larvae in relation to effluent plumes from sewage outfalls and depth of water. Mar Biol 1 13:549-559

Grimes CB, Finucane JH (1991) Spatial distribution and abundance of larval and juvenile fish chlorophyll and macrozooplankton around the Mississippi River discharge plume and the role of the plume in fish recruitment. Mar Ecol Prog Ser 75:109-119

Haney JF (1988) Diel patterns of zooplankton behaviour. Bull Mar Sci 43:583-603

Hardy JT (1982) The sea surface microlayer biology, chemistry, and anthropogenic enrichment. Prog Oceanogr 11: $307-328$

Hardy JT, Apts CW. Crecelius EA, Bloom NS (1985) Sea-surface microlayer metals enrichment in an urban and rural bay. Estuar Coast Shelf Sci 20:299-312

Hardy JT, Crecelius EA, Antrim L, Broadhurst VL, Apts CW, Gurtisen JM, Fortman TJ (1987a) The sea-surface microlayer of Puget Sound: Part 2. Concentrations of contaminants and relation to toxicity. Mar Environ Res 23 $251-271$

Hardy JT, Kiesser S, Antrim L, Stubin A, Kocan R, Strand J (1987b) The sea-surface microlayer of Puget Sound: Part 1 Toxic effects on fish eggs and Iarvae. Mar Environ Res 23:227-249

Haury IR, McGowan JA, Welbe PH (1978) Patterns and processes in the time-space scales of plankton distributions. In: Steele J (ed) Spatial pattern in plankton communities. Plenum Press, New York, p 277-337

Hunter JR, Mitchell CT (1967) Association of fishes with flotsam in the offshore waters of Central America. Fish Bull US 66:13-29

Karas P, Neuman E. Sandstrom O (1991) Effects of a pulp mill effluent on the population dynamics of perch Perca lluviatilis. Can J Fish Aquat Sci 48:28-34
Kendall AW, Naplin NA (1981) Diel-depth distribution of summer ichthyoplankton in the Middle Atlantic Bight. Fish Bull US 79:705-726

Keough MJ, Black KP (1996) Predicting the scale of marine impacts: understanding planktonic links between populations. In: Schmitt RJ, Osenberg CW (eds) Detecting ecological impacts: concepts and applications in coastal habitats. Academic Press, San Diego, p 199-234

Kingsford MJ (1990) Linear oceanographic features: a focus for research on recruitment processes. Aust J Ecol 15: $391-401$

Kingsford MJ (1992) Drift algae and small fish in coastal waters of northeastern New Zealand. Mar Ecol Prog Ser 80:41-55

Kingsford MJ (1993) Biotic and abiotic structure in the pelagic environment: importance to small fishes. Bul1 Mar Scl 53 $393-415$

Kingsford MJ, Choat JH (1986) The influence of surface slicks on the distribution and movements of small fish. Mar Biol 91:161-171

Kingsford MJ, Gray CA (1996) Influence of pollutants and oceanography on abundance and deformities of wild fish larvae. In: Schmitt RJ, Osemberg CW (cds) Detecting ecological impacts: concepts and applications in coastal habitats. Academic Press, San Diego, p 235-255

Kingsford MJ, Suthers IM (1994) Dynamic estuarine plumes and fronts: importance to small fish and plankton in coastal waters of NSW, Australia. Cont Shelf Res 14 : $655-672$

Kingsford MJ, Wolanski E, Choat JH (1991) Influence of tidally induced fronts and Langmuir circulations on distribution and movements of presettlement fishes around a coral reef. Mar Biol 109:167-190

Le Fèvre J (1986) Aspects of the biology of frontal systems. Adv Mar Biol 23:163-299

Lobel PS, Robinson AR (1988) Larval fishes and zooplankton in a cyclonic eddy in Hawaiian waters. J Plankton Res 10 : $1209-1223$

Longwell AC, Chang S, Hebert A, Hughes JB, Perry D (1992) Pollution and development abnormalities of Atlantic fishes. Environ Biol Fish 35:1-21

Mackas DL, Louttit GC (1988) Aggregation of the copepod Neocalanus plumchrus at the margin of the Fraser River plume in the Strait of Georgia. Bull Mar Sci 43:810-824

MacLean SA (1993) Pathological conditions of Narragansett Bay young-of-the-year winter flounder. Am Fish Soc Symp $14: 47-54$

Mitchell CT, Hunter JR (1970) Fishes associated with drifting kelp Macrocystis pyrifera off the coast of southern California and northern Baja California. Calif Fish Game 56 $288-297$

Murdoch RC (1989) The effects of a headland eddy on surface macro-zooplankton assemblages north of Otago peninsula, New Zealand. Estuar Coast Shelf Sci 29:361-383

Nair VR, Gajbhiye SN, Desai BN (1991) Effect of pollution on the distribution of chaetognaths in the nearshore waters of Bombay. Ind J Mar Sci 20:43-48

Neilson JD, Perry RI (1990) Diel vertical migrations of marine fishes: an obligate or facultative process? Adv Mar Biol 26 : $115-168$

Norcross BL, Shaw RF (1984) Oceanic and estuarine transport of fish eggs and larvae: a review. Trans Am Fish Soc 113: $153-165$

Otway NM, Gray CA, Craig JR, MCVea TA, Ling JE (1996) Assessing the impacts of deepwater sewage outfalls on spatially and temporally variable marine communities. Mar Environ Res 41:45-71 
Owen RW (1981) Fronts and eddies in the sea: mechanisms interactions and biological effects. In: Longhurst AR (ed) Analysis of marine ecosystems. Academic Press, London, p $197-233$

Raimondi PT, Reed DC (1996) Determining the extent of ecological impacts caused by local anthropogenic disturbances in coastal manne habitats. In: Schmitt RJ, Osenberg CW (eds) Detecting ecological impacts: concepts and applications in coastal habitats. Academic Press, San Diego, p $179-198$

Rosenthal H, Alderdice DF (1976) Sublethal effects of environmental stressors natural and pollutional on marine fish eggs and larvae. J Fish Res Bd Can 33:2047-2065

Sabates A (1990) Changes in the heterogeneity of mesoscale distribution patterns of larval fish associated with a shallow coastal haline front. Estuar Coast Shelf Sci 30: $131-140$

Safran P, Omori M (1990) Some ecological observations on fishes associated with drifting seaweed of Tohoku coast, Japan. Mar Biol 105:395-402

Shanks AL (1983) Surface slicks associated with tidally forced internal waves may transport pelagic larvae of benthic invertebrates and fishes shoreward. Mar Ecol Prog Ser 13: $311-315$

Shanks AL (1985) Behavioural basis of internal-wave-induced

This article was submitted to the editor shoreward transport of megalopae of the crab Pachygrapsus crassipes. Mar Ecol Prog Ser 24:289-295

Tanabe S, Nishimura A, Hanaoka S, Yanagi T, Takeoka $H$, Tatsukawa R (1991) Persistant organochlorines in coastal fronts. Mar Pollut Bull 22:344-353

Thorrold SR, Shenker JM, Wishinski E, Mojica R, Maddox ED (1994) Larval supply of shorefishes to nursery habitats around Lee Stocking Island, Bahamas. 1. Small-scale distribution patterns. Mar Biol 118:555-566

Underwood AJ (1981) Techniques of analysis of variance in experimental marine biology and ecology. Oceanogr Mar Biol A. Rev 19:513-605

Underwood AJ, Fairweather PG (1989) Supply-side ecology and benthic marine assemblages. Trends Ecol Evol 4:16-20

Weis P, Weis JS (1989) Effects of environmental pollutants on early fish development. Rev Aquat Sci 1:45-73

Westernhagen HV (1988) Sublethal effects of pollutants on fish eggs and larvae. In: Hoar WS, Randall DJ (eds) Fish physiology XI. Academic Press, San Diego, p 253-346

Wolanski E, Hamner WM (1988) Topographically controlled fronts in the ocean and their biological influence. Science $241: 177-181$

Zeldis JR, Jillett JB (1982) Aggregation of pelagic Munida gregaria (Fabricus) (Decapoda Anomura) by coastal fronts and internal waves. J Plankton Res 4:839-857

Manuscript first received: July 26, 1995

Revised version accepted: April 9, 1996 